\title{
Mesenchymal stem cells ameliorate partial bladder outlet obstruction-induced epithelial- mesenchymal transition type II independent of mast cell recruitment and degranulation
}

\author{
Rutuja Kadam; Bridget Wiafe; Peter D. Metcalfe \\ ${ }^{1}$ Department of Surgery, Faculty of Medicine and Dentistry, University of Alberta, Edmonton, AB, Canada
}

Cite as: Can Urol Assoc J 2020 July 17; Epub ahead of print. http://dx.doi.org/10.5489/cuaj.6501

Published online July 17, 2020

$* * *$

\section{Abstract}

Introduction: Partial bladder outlet obstruction (pBOO) results in increased urinary storage pressure and significant morbidity. Increased pressure results in a sequence of programmed events: an initial inflammatory phase, smooth muscle hypertrophy, and fibrosis. Although epithelial-mesenchymal transition (EMT) and mast cell accumulation play intermediary roles in some fibrotic conditions, their role in $\mathrm{pBOO}$ has not yet been elucidated. Mesenchymal stem cell (MSC) therapy is emerging as a promising treatment for several conditions. It potently inhibits bladder deterioration after pBOO; however, its mechanism of action is insufficiently understood. Thus, we hypothesize that EMT type II pathway plays a significant role in $\mathrm{pBOO}$, aided by the recruitment and activation of mast cells, and these are potently inhibited by MSCs.

Methods: PBOO was surgically induced in female Sprague-Dawley rats and simultaneously treated with MSCs. Treatment effect was determined after two or four weeks, and compared to untreated controls. Immunohistochemistry was used to measure markers characteristic of EMT (vimentin, collagenase, and collagen). Whole and degranulated mast cell counts were also performed.

Results: PBOO resulted in an increased expression of collagenase, vimentin, and collagen. Mast cell recruitment increased proportionately to the length of bladder obstruction. MSC treatment significantly mitigated the EMT type II response, but mast cell recruitment and degranulation were unaffected.

Conclusions: Our results demonstrate the involvement of EMT type II in the pathophysiology of pBOO and confirm its mitigation with MSC treatment independent of mast cells response. The observations provide insight into the mechanism of action and have therapeutic ramifications. 


\section{Introduction}

Partial bladder outlet obstruction (pBOO) is a ubiquitous condition in urology, with etiology ranging from prostatic hyperplasia in elderly males to posterior valves in the fetus. Several studies have promoted the idea that following $\mathrm{pBOO}$, the bladder detrusor muscle generates a high-pressure state, causing programmed progressive deterioration of the bladder with accompanying biochemical and molecular changes. An initial inflammatory phase gives rise to bladder smooth muscle hypertrophy, followed by compensatory angiogenesis, infiltration of the extracellular space with collagen and resultant decompensatory bladder fibrosis. ${ }^{1,2}$ However, despite this widespread recognition, current treatment modalities for $\mathrm{pBOO}$ remain either crude or inadequate. Neither clean intermittent catheterization nor oral anticholinergics target the physiologic pathways to inhibit the progression of this pathology. This gap necessitates the use of a bimodal therapy that attenuates the pathological processes underlying $\mathrm{pBOO}$, and at the same time, can promote regeneration of healthy bladder tissue.

Over recent years, Mesenchymal Stem/Stromal Cells (MSCs) have propelled to the forefront as a promising treatment for inflammatory and fibrotic diseases. MSCs are multipotent cells, most commonly isolated for clinical use from adults from the bone marrow and adipose tissue. ${ }^{3}$ MSCs possess a lack of immunogenicity and can modulate immune responses making them efficacious in the treatment of rheumatoid arthritis, and graft versus host disease. ${ }^{4,5}$ Alongside these properties, the paracrine effects of MSCs have been shown to attenuate fibrosis in the liver, lungs, and kidneys. ${ }^{6,7,8}$ Concerning $\mathrm{pBOO}$, we have previously demonstrated the anti-inflammatory and anti-fibrotic effects of MSC treatment in-vitro using hypoxic bladder smooth muscle cells, and in-vivo using acute and long-term pBOO rat models. ${ }^{9,}{ }^{10,11}$ Since MSC therapy for $\mathrm{pBOO}$ holds great promise, it necessitates a fuller understanding of the underlying mechanisms that drive $\mathrm{pBOO}$ and the effect that MSCs have on them.

Type 2 Epithelial to Mesenchymal Transition (EMT Type II) is a cellular process that can generate aberrant myofibroblasts whose fibrotic activity has adverse effects on organ function. ${ }^{12}$ The process is a double-edged sword that is initially beneficial in wound healing; however, in cases of prolonged inflammation, it results in excessive extracellular matrix deposition and fibrosis. ${ }^{13}$ During EMT Type II, cells lose their typical epithelial architecture, apical-basal polarity and instead gain migratory abilities, a spindle-shaped mesenchymal phenotype, and express vimentin - a type III intermediate filament. ${ }^{12,14}$ Transforming Growth Factor-Beta (TGF$\beta$ ), a pleiotropic pro-fibrotic factor, is critical to this process, and acts via downstream phosphorylation of mothers against decapentaplegic homolog 2/3 (SMAD2/3), leading to eventual myofibroblast differentiation. ${ }^{15,16}$ Collagenases can degrade extracellular matrix components ${ }^{17}$ and promote pericellular collagenolysis to facilitate cell migration and invasion. ${ }^{18,19}$

Mast cells are multi-functional immune cells that originate in the bone marrow as naïve progenitor cells, are recruited via chemotactic inflammatory cytokines, and mature upon penetrating tissue. ${ }^{20}$ The hallmark of their activation involves degranulation and secretion of 
cytokines such as TGF- $\beta 1$ and chymase, which promote fibrosis. ${ }^{21,}{ }^{22}$ Since MSCs have an immunomodulatory effect, they have the potential to alter mast cell accumulation and degranulation and inhibit their deleterious effects.

The role of EMT Type II, within the context of pBOO, has not yet been elucidated. Thus, we hypothesize that the EMT Type II pathway is a primary pathway through which $\mathrm{pBOO}$ results in fibrosis, and that mast cells play a prominent role in this process. MSCs achieve their therapeutic, anti-fibrotic effects by attenuating these processes.

\section{Methods}

\section{Animal and study design}

All protocols were approved by the University of Alberta Ethics Committee for the Care and Use of Experimental Animals. 20 Healthy adult female Sprague Dawley rats (approximately 250$300 \mathrm{~g}$ ) were randomly assigned to 5 groups as follows: a) obstructed for 2 weeks (pBOO2 weeks), b) obstructed for 4 weeks (pBOO-4weeks), c) obstructed with MSC therapy for 2 weeks (pBOO+MSCs-2weeks), d) obstructed with MSC therapy for 4 weeks (pBOO+MSCs4 weeks), and e) unobstructed controls ( $\mathrm{n}=4$ in each group). The animals resided two per cage with free access to food and water.

\section{Induction of $\mathrm{pBOO}$}

Rats in $\mathrm{pBOO}$ groups underwent urethral ligation under isoflurane anesthesia to create partial bladder outlet obstruction as described earlier. ${ }^{2,11}$ In sum, the bladder was exposed, and an 18gauge angiocatheter was inserted into the urethra. A 2-0 silk suture was then tied around the urethra, and the catheter was removed. All operated rats were provided with cotrimoxazole-water and maintained on meloxicam $(1 \mathrm{mg} / \mathrm{kg})$ and buprenorphine $(0.02 \mathrm{mg} / \mathrm{kg})$ to manage pain.

\section{Mesenchymal stem cell treatment}

Immediately after pBOO, the therapy groups were given a tail vein injection of Sprague Dawley bone-marrow-derived MSCs commercially obtained from Cyagen Biosciences Inc, (Sunnyvale, CA) in 2015. The cells obtained were characterized by the supplier to be positive for CD90, CD44, CD29 and negative for CD34, CD45, CD11b/c by flow cytometry analysis. They also tested negative for bacteria, fungi, and mycoplasma. The MSCs were cultured to expand in alpha-minimal essential medium ( $\alpha$-MEM) supplemented with 10\% heat-inactivated fetal bovine serum, L-glutamine $(290 \mu \mathrm{g} / \mathrm{ml})$, 4-(2-hydroxyethyl)-1-piperazineethanesulfonic acid (HEPES) $(100 \mathrm{mM})$, sodium pyruvate $(1 \mathrm{mM})$, penicillin-streptomycin $(100 \mathrm{U} / \mathrm{ml})$ (Life Technologies, Burlington, Canada), and $5 \mathrm{ng} / \mathrm{ml}$ fibroblast growth factor-2 (FGF-2) (Neuromics, Edina, MN, USA). MSCs between passages 2 and eight were used for this experiment. One million stem cells in the log phase of growth per $0.5 \mathrm{ml}$ PBS were injected per animal. At the experimental endpoint, bladder tissue was harvested and part fixed in $4 \%$ Paraformaldehyde for immunohistochemistry studies. 


\section{Antibodies}

Mouse monoclonal antibodies against collagenase (DSHB H18G8), and vimentin (DSHB 3CB2) deposited by Werb, Z. and De La Rosa, E.J. respectively were obtained from the Developmental Studies Hybridoma Bank, created by the NICHD of the NIH and maintained at The University of Iowa, Department of Biology, Iowa City, IA 52242. A secondary horseradish peroxidaseconjugated antibody rabbit anti-mouse (abcam6728) was purchased from the indicated supplier.

\section{Immunohistochemistry studies}

Formalin-fixed bladder tissues were embedded in paraffin blocks and sectioned into $5 \mu \mathrm{m}$ thickness. Then, the sections were deparaffinized by one-hour incubation at $65^{\circ} \mathrm{C}$, followed by two changes of xylene and rehydration in decreasing concentrations of alcohol and water. Samples underwent heat-mediated antigen retrieval in sodium citrate solution, followed by blocking of endogenous peroxidase activity in a solution of methanol and hydrogen peroxide. 5\% BSA was used to block the tissues for an hour and a half. Overnight incubation with a primary antibody concentration of 1:100 vimentin and 1:45 collagenase in 2\% BSA was performed. The following day, washes were followed by incubation in 1:200 secondary antibody in TBST for an hour and a half. Following subsequent washes, the immunoreaction was visualized with 3'3'diaminobenzidine (DAB Sigma D8001). Tissues were counterstained with Mayer's hematoxylin (Sigma MHS32), dehydrated in 100\% ethanol, cleared in xylene and mounted for microscopic visualization. Microscopic images were taken using an Eclipse Ti-S microscope (Nikon Canada, Mississauga, Canada).

\section{Toluidine blue tissue staining and mast cell degranulation count}

Toluidine blue stock solution was prepared from powder (Sigma 198161) diluted in 70\% ethanol. At the time of the experiment, a working solution was prepared by combining the stock solution with $1 \% \mathrm{NaCl}$ with a $\mathrm{pH}$ of 2.3 . Following deparaffinization at $65^{\circ} \mathrm{C}$, xylene clearance and rehydration, as indicated by steps outlined earlier, slides were stained in toluidine blue working solution for 2-3 minutes. Next, slides were washed in distilled water, dehydrated in $100 \%$ ethanol, cleared in xylene and mounted. Microscopic images were taken using an Eclipse Ti-S microscope (Nikon Canada, Mississauga, Canada). Images at a higher magnification were taken using a Zeiss Axio Imager Microscope with 20x 1.3 NA Plan Apocromat lens and captured with Infinity3-3UR camera with Infinity analyze and capture software for windows.

A mast cell degranulation count was performed with an Eclipise Ti-S light microscope by two independent observers. Per tissue, mast cells in 100 high power fields were counted. Whole non-degranulated mast cells which had an intact, uniform, dark violet-indigo colour with little or no space visible inside them were counted per field. The number of degranulated mast cells and their extracellular granules which exhibited a lighter purple colour, were also noted. 


\section{Trichrome staining}

Deparaffinised bladder tissues were incubated in Bouin's solution, washed and stained using Gomori's stain for 15 minutes. Differentiation was performed in $0.5 \%$ acetic acid for 2 minutes and washed sections were dehydrated, cleared in xylene and mounted. Microscoptic images were captured using Eclipse Ti-S microscope.

\section{Results}

\section{EMT type II response}

Vimentin, a marker of the pervasiveness of EMT, increased with obstruction. The protein was immunolocalized to the detrusor after 2 and four weeks of obstruction. However, MSC treatment led to a significant decrease in its expression (Figure 1a). Similarly, collagenase expression increased with obstruction time and was prevalent in the lamina propria as well as the detrusor. MSC treatment attenuated the expression of these proteins at the same time points (Figure 1b).

\section{Mast cell response}

Mast cell accumulation in the bladder tissue increased with obstruction but remained unchanged with MSC treatment. Even though the mast cells were broadly distributed throughout the layers of the bladder, there appeared to be more in the suburothelial layer, and around blood vessels (Figure 2). Increase in the number of mast cells was directly proportional to the obstruction time, and degranulated mast cells increased in accordance. Nonetheless, treatment with MSCs neither affected the total mast cell count nor the proportion of degranulation (Figure 3).

\section{Collagen accumulation response}

As revealed by Trichrome staining, $\mathrm{pBOO}$ groups displayed an increase in collagen deposition in the lamina propria and intra-detrusor spaces. This collagen infiltration of the bladder smooth muscles was heightened in 4-week obstructed bladders, resulting in the observed distorted histology. However, MSC injection resulted in the inhibition of connective tissue infiltration at the same time points, and the treated groups displayed normal bladder histology (Figure 4).

\section{Discussion}

EMT Type II is a critical process in the physiology of inflammation; however, when unchecked, it can result in pathologic fibrosis. We have previously shown that $\mathrm{pBOO}$ results in fibrosis and MSCs can inhibit this. In this investigation, we wished to more pointedly explore the role of EMT and mast cells in pBOO. We demonstrated the expression of EMT markers and their inhibition by MSC treatment. This study reveals that activation of the EMT Type II program does play a role in achieving the fibrotic pathophysiology of $\mathrm{pBOO}$, thus supporting our hypothesis.

Our previous investigation using the same animal population demonstrated that obstruction upregulates the gene expression of TGF- $\beta 1$ - a master-inducer capable of sustaining 
the entire EMT process. ${ }^{11}$ Additionally, our lab previously confirmed the transcriptional upregulation of SMAD2/3 alongside increased protein expression of phosphorylated SMAD2/3 in bladder urothelium after $\mathrm{pBOO} .{ }^{11}$ The TGF- $\beta 1$-SMAD2/3 pathway is closely tied to myofibroblast differentiation ${ }^{16}$ and EMT. ${ }^{15}$ Specifically, the phosphorylated SMAD2/3 combine with SMAD4 to form a heterotrimer complex which translocates to the nucleus to regulate gene expression and generate myofibroblasts. ${ }^{23}$ Given the well-established relationship between EMT and myofibroblast activation, the pBOO-induced EMT in the current study may result in the concomitant increase in myofibroblasts accumulation leading to fibrosis. Collagenases play an important role in cell invasion and migration through pericellular collagenolysis. ${ }^{18,19}$ One characteristic feature of $\mathrm{pBOO}$ is the increased deposition of collagen. In this study, we also found a copious amount of collagen deposition in the lamina propria and detrusor with increased obstruction time. Results of the current study show the activation of EMT Type II sustains the pathway of this disease. Thus, the concurrent increase in protein expression of vimentin, collagenase, and collagen, confirms the contributive role of EMT Type II in pBOO-induced bladder fibrosis via the TGF- $\beta 1$-SMAD2/3 pathway.

In keeping with current findings, EMT Type II results in a simultaneous increase in mast cell accumulation and associated degranulation. A study conducted by Michishita et al. ${ }^{24}$ also found an upregulation of mast cells when examining $\mathrm{pBOO}$ in a rat model at one-week of obstruction. The inflammatory bladder disease - bladder pain syndrome/interstitial cystitis (BPS/IC) is the most common cause of mast cell recruitment. ${ }^{25}$ Previous studies have implicated mast cells in fibrosis since they are capable of secretion and chymase-mediated activation of TGF- $\beta 1$ and hence the induction of the pathophysiology of fibrotic diseases, ${ }^{21,22}$ as noted with renal fibrosis after ureteric obstruction. ${ }^{26}$ Thus, in this study, mast cells serve as a twofold marker for inflammation as well as the consequent EMT process. The increased mast cell count with obstruction demonstrates increased inflammation of the bladder after $\mathrm{pBOO}$. In our previous investigation, we demonstrated that the gene transcription of TNF-alpha increased in obstructed bladders. ${ }^{11} \mathrm{TNF}-\alpha$ is a potent chemoattractant for mast cells and could hence be the mechanism underlying the observed mast cell accumulation in the current study. ${ }^{20} \mathrm{We}$ were also able to demonstrate that $\mathrm{pBOO}$ results in the associated degranulation of mast cells. Therefore, the inflammatory process is integral to mast cell recruitment and activation.

MSCs inhibit EMT Type II without affecting mast cell accumulation and degranulation. Since our previous work demonstrated that MSCs downregulate TGF- $\beta 1$ and SMAD2/3, and since the EMT Type II process canonically occurs via the signaling of stated cytokines, it is likely that their inhibition is the primary cause behind the ensuing decrease in myofibroblasts, collagenase, and collagen expression. Regarding the unaffected mast cell accumulation and degranulation, a few possible explanations are possible. Firstly, MSCs may likely exert their effects by interacting with other sources of pro-fibrotic mediators, such as the macrophage and T-helper cells. ${ }^{27}$ The effect of MSCs on these additional components of the immune system deserves further investigation. Secondly, the effect of MSCs on mast cells could be a function of 
time, better observed after a more extended period than the four weeks researched in this study. Lastly, mast cell mediators initially play a role in wound healing, a process which goes awry with chronic inflammation and leads to fibrosis. Thus, perhaps this mechanism is conserved by MSCs due to its initially advantageous protective purpose, and MSCs instead downregulate cytokines like TGF- $\beta 1$ and SMAD2/3 that are more proximally related to fibrosis. Despite having no significant effect on mast cells, MSC treatment has global benefit, as our previous work has shown a predominant decrease in inflammation, smooth muscle cell hypertrophy, fibrosis, and most importantly, improvement in urodynamic parameters. ${ }^{10,11}$

We wish to acknowledge a few of the limitations of this study. This manuscript is designed as a descriptive, qualitative, assessment of the role of EMT in bladder fibrosis. We were unable to perform quantitative (RT-PCR, ELISA) experiments because of limited quantities of tissue available. However, we feel confident that our previous studies have robustly confirmed the degree of inflammation and fibrosis. ${ }^{2,9,10,11}$ Therefore, our descriptive exploration of EMT as a mechanism towards this is justified as a means of describing the pathophysiology, and one mechanism by which MSC exert their protective effect.

Our model is designed to resemble clinical conditions such as posterior urethral valves, neurogenic bladders with detrusor sphincter dyssynergia, and prostatic hypertrophy. We have delineated many biochemical pathways and means of inhibition, however, acknowledge multiple shortcomings. The acute severity of obstruction and timing of therapeutic administration is less relevant to clinical practice. No human receives treatment the minute an acute, nearly complete obstruction occurs. However, we have been using MSC treatment at the time of surgical induction of $\mathrm{pBOO}$, as this has given us the most efficacious means of mitigating the inflammatory response and preventing detrusor deterioration. Because our injections at the time of pBOO induction have been so effective, we have not felt the need to repeat them. But, instead have begun working on experiments to confirm the efficacy of treatment after $\mathrm{pBOO}$ and once bladder changes have occurred. We will give the MSC 2 and 4 weeks after $\mathrm{pBOO}$ and determine its effectiveness at repairing damaged tissue, instead of preventing the damage.

\section{Conclusions}

Our finding that $\mathrm{pBOO}$-induced fibrosis occurs via the EMT Type II pathway reinforces its similarities with other fibrotic pathologies. We suspect that $\mathrm{pBOO}$ fibrosis is liable to similar mechanisms that affect such diseases as cirrhosis, renal fibrosis, and pulmonary fibrosis wherein EMT Type II pathway is demonstrably involved. We look forward to future studies, to determine the efficacy of MSC treatment in a delayed fashion, with a pre-established EMT pathway, to determine if MSCs can reverse this pathophysiology.

\section{References}


1. Fusco F, Creta M, De Nunzio C, et al. Progressive bladder remodeling due to bladder outlet obstruction: a systematic review of morphological and molecular evidences in humans. BMC Urol 2018;18(1):15.

2. Metcalfe PD, Wang J, Jiao H, et al. Bladder outlet obstruction: progression from inflammation to fibrosis. BJU Int 2010;106(11):1686-94.

3. Fitzsimmons RE, Mazurek MS, Soos A, et al. Mesenchymal Stromal/Stem Cells in Regenerative Medicine and Tissue Engineering. Stem Cells Int 2018:1-16.

4. Lee K, Park N, Jung H, et al. Mesenchymal stem cells ameliorate experimental arthritis via expression of interleukin-1 receptor antagonist. PloS One 2018;13(2):e0193086.

5. Zhao K, Lou R, Huang F, et al. Immunomodulation Effects of Mesenchymal Stromal Cells on Acute Graft-versus-Host Disease after Hematopoietic Stem Cell Transplantation. Biol Blood Marrow Transplant 2015;21(1):97-104.

6. Fu X, Jiang B, Zheng B, et al. Heterogenic transplantation of bone marrow-derived rhesus macaque mesenchymal stem cells ameliorates liver fibrosis induced by carbon tetrachloride in mouse. PeerJ 2018;6:e4336.

7. Li X, Wang Y, An G, et al. Bone marrow mesenchymal stem cells attenuate silicainduced pulmonary fibrosis via paracrine mechanisms. Toxicol Lett 2017;270:96-107.

8. Lv S, Liu G, Sun A, et al. Mesenchymal stem cells ameliorate diabetic glomerular fibrosis in vivo and in vitro by inhibiting TGF- $\beta$ signalling via secretion of bone morphogenetic protein 7. Diab Vasc Dis Res 2014;11(4):251-61.

9. Al-Saikan B, Ding J, Tredget E, et al. Benefits of mesenchymal stem cells after partial bladder outlet obstruction. Can Urol Assoc J 2016;10(1-2):1.

10. Wiafe B, Adesida A, Churchill T, et al. Mesenchymal stem cells inhibit hypoxia-induced inflammatory and fibrotic pathways in bladder smooth muscle cells. World $\mathrm{J}$ Urol 2018;36(7):1157-65.

11. Wiafe B, Adesida A, Churchill T, et al. Mesenchymal stem cell therapy inhibited inflammatory and profibrotic pathways induced by partial bladder outlet obstruction and prevented high-pressure urine storage. J Pediatr Urol 2019;15(3):254.e1-254.e10.

12. Kalluri R, Weinberg RA. The basics of epithelial-mesenchymal transition. J Clin Invest 2009;119(6):1420-28.

13. Tennakoon A, Izawa T, Kuwamura M, et al. Pathogenesis of Type 2 Epithelial to Mesenchymal Transition (EMT) in Renal and Hepatic Fibrosis. J Clin Med 2015;5(1):4.

14. Mendez MG, Kojima S, Goldman RD. Vimentin induces changes in cell shape, motility, and adhesion during the epithelial to mesenchymal transition. FASEB J 2010;24(6):1838-51.

15. Bani-Hani AH, Campbell MT, Meldrum DR, et al. Cytokines in EpithelialMesenchymal Transition: A New Insight into Obstructive Nephropathy. J Urol 2008;180(2):461-68. 
16. Gu L, Zhu Y, Yang X, et al. Effect of TGF- $\beta /$ Smad signaling pathway on lung myofibroblast differentiation. Acta Pharmacol. Sin. 2007;28(3):382-391.

17. Somerville RP, Oblander SA, Apte SS. Matrix metalloproteinases: old dogs with new tricks. Genome Biol 2003;4(6):216.

18. Itoh Y, Seiki M. MT1-MMP: A potent modifier of pericellular microenvironment. J Cell Physiol 2006;206(1): 1-8.

19. Sabeh F, Shimizu-Hirota R, Weiss SJ. Protease-dependent versus -independent cancer cell invasion programs: three-dimentional amoeboid movement revisited. J Cell Biol 2009;185(1):11-9.

20. Brzezińska-Błaszczyk E, Pietrzak A, Misiak-Tłoczek AH. Tumor Necrosis Factor (TNF) Is a Potent Rat Mast Cell Chemoattractant. J Interferon Cytokine Res 2007;27(11):91120.

21. Chen H, Xu Y, Yang G, et al. Mast cell chymase promotes hypertrophic scar fibroblast proliferation and collagen synthesis by activating TGF- $\beta 1 /$ Smads signaling pathway. Exp Ther Med 2017;14(5):4438-42.

22. Kanbe N, Kurosawa M, Nagata H, et al. Cord blood-derived human cultured mast cells produce transforming growth factor $\beta 1$. Clin Exp Allergy 1999;29(1):105-13.

23. Khalil H, Kanisicak O, Prasad V, et al. Fibroblast-specific TGF- $\beta-S m a d 2 / 3$ signaling underlies cardiac fibrosis. J Clin Invest 2017;127(10):3770-83.

24. Michishita M, Tomita K, Yano K, et al. Mast Cell Accumulation and Degranulation in Rat Bladder with Partial Outlet Obstruction. Adv Ther 2015;32(S1):16-28.

25. Van de Merwe JP, Nordling J, Bouchelouche P, et al. Diagnostic Criteria, Classification, and Nomenclature for Painful Bladder Syndrome/Interstitial Cystitis: An ESSIC Proposal. Eur Urol 2008;53(1):60-7.

26. Summers SA, Gan P, Dewage L, et al. Mast cell activation and degranulation promotes renal fibrosis in experimental unilateral ureteric obstruction. Kidney Int 2012;82(6):67685.

27. Lupher ML, Gallatin WM. Regulation of Fibrosis by the Immune System. Adv Immunol 2006; 89:245-88. 


\section{Figures and Tables}

Fig. 1. Increased vimentin and collagenase expression and activity in partial bladder outlet obstruction (pBOO) was inhibited by mesenchymal stem cells (MSCs). (A) Vimentin protein expression progressively increased with $\mathrm{pBOO}$ duration and decreased significantly with MSC treatment to normal expression. (B) Collagenase protein levels, also identified in brown staining, increased significantly in the lamina propria and detrusor after pBOO; however, collagenase levels were downregulated in MSC groups. Representative micrographs were captured at 10x magnification, and scale bars represent $100 \mu \mathrm{m}$.

A

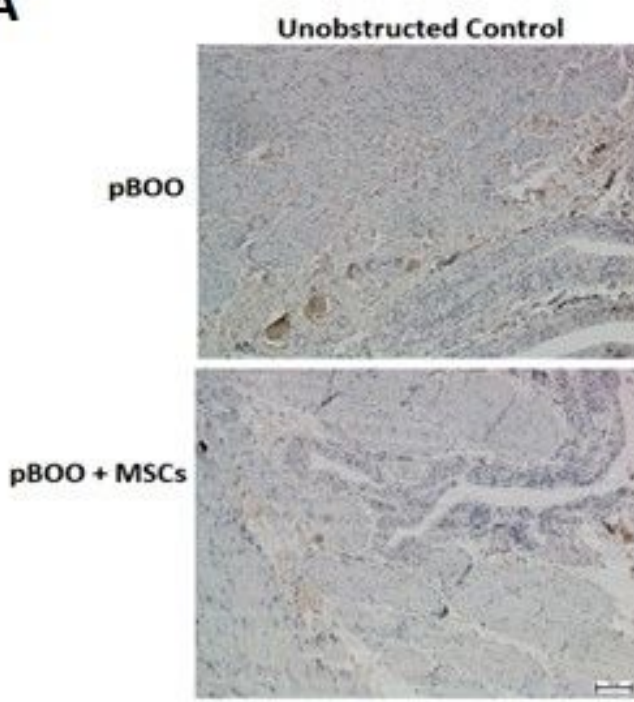

Vimentin Protein

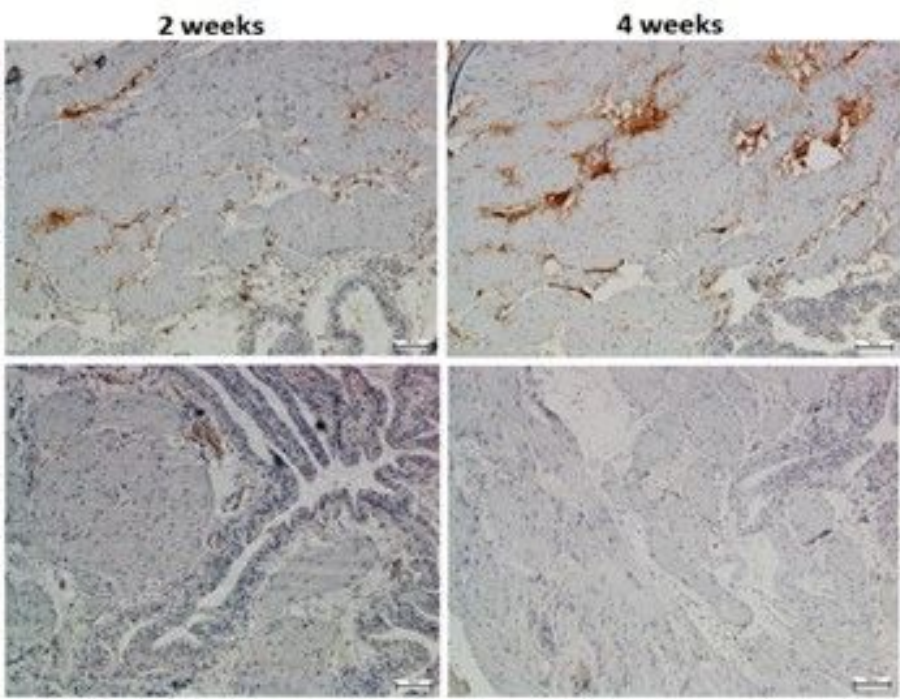

B

Collagenase Protein

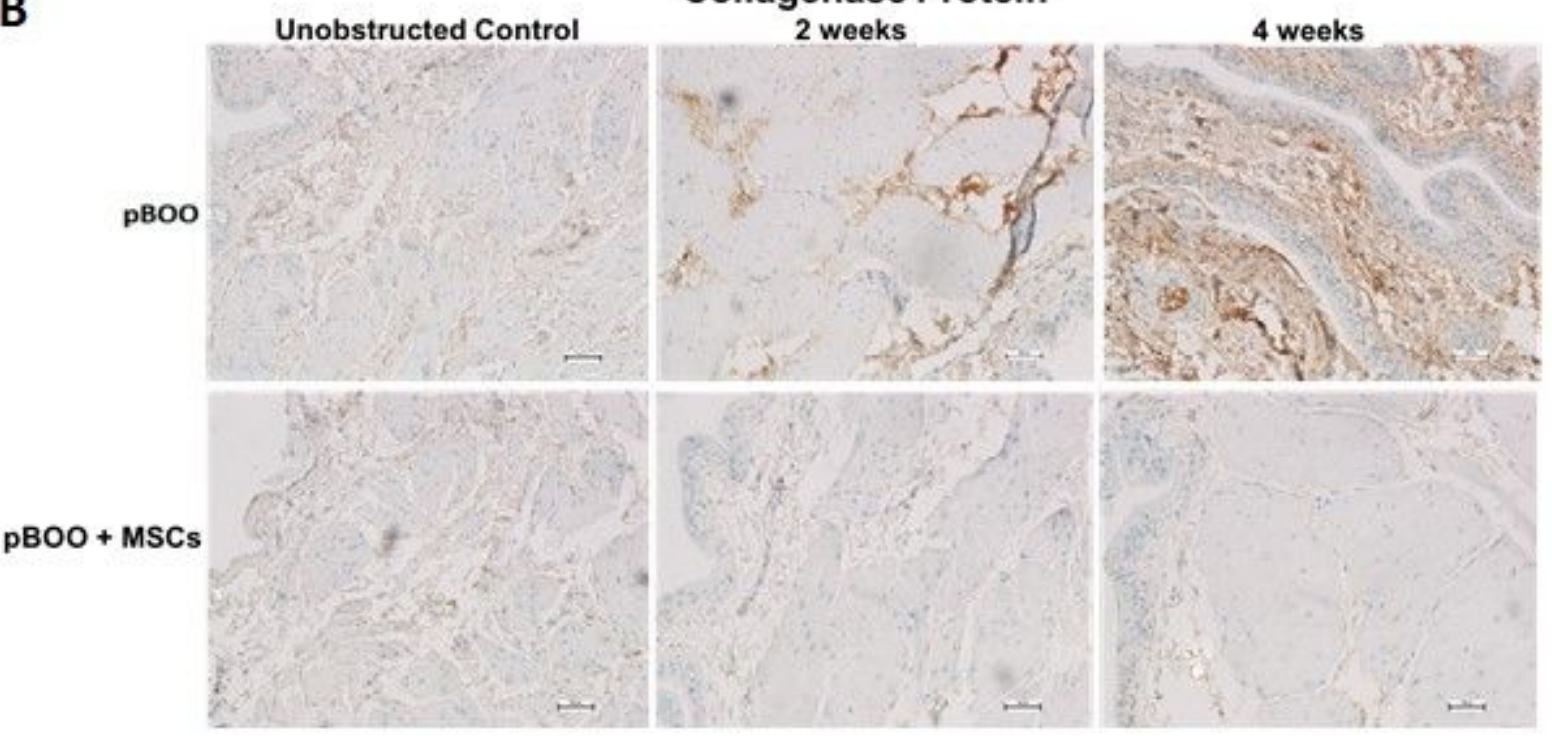


Fig. 2. Mast cell accumulation increased with obstruction and was unaffected by mesenchymal stem cells (MSCs). Mast seen as dark blue cells, were broadly distributed throughout the bladder layers. Representative micrographs were captured at 10x magnification, and scale bars represent $100 \mu \mathrm{m}$. pBOO: partial bladder outlet obstruction.

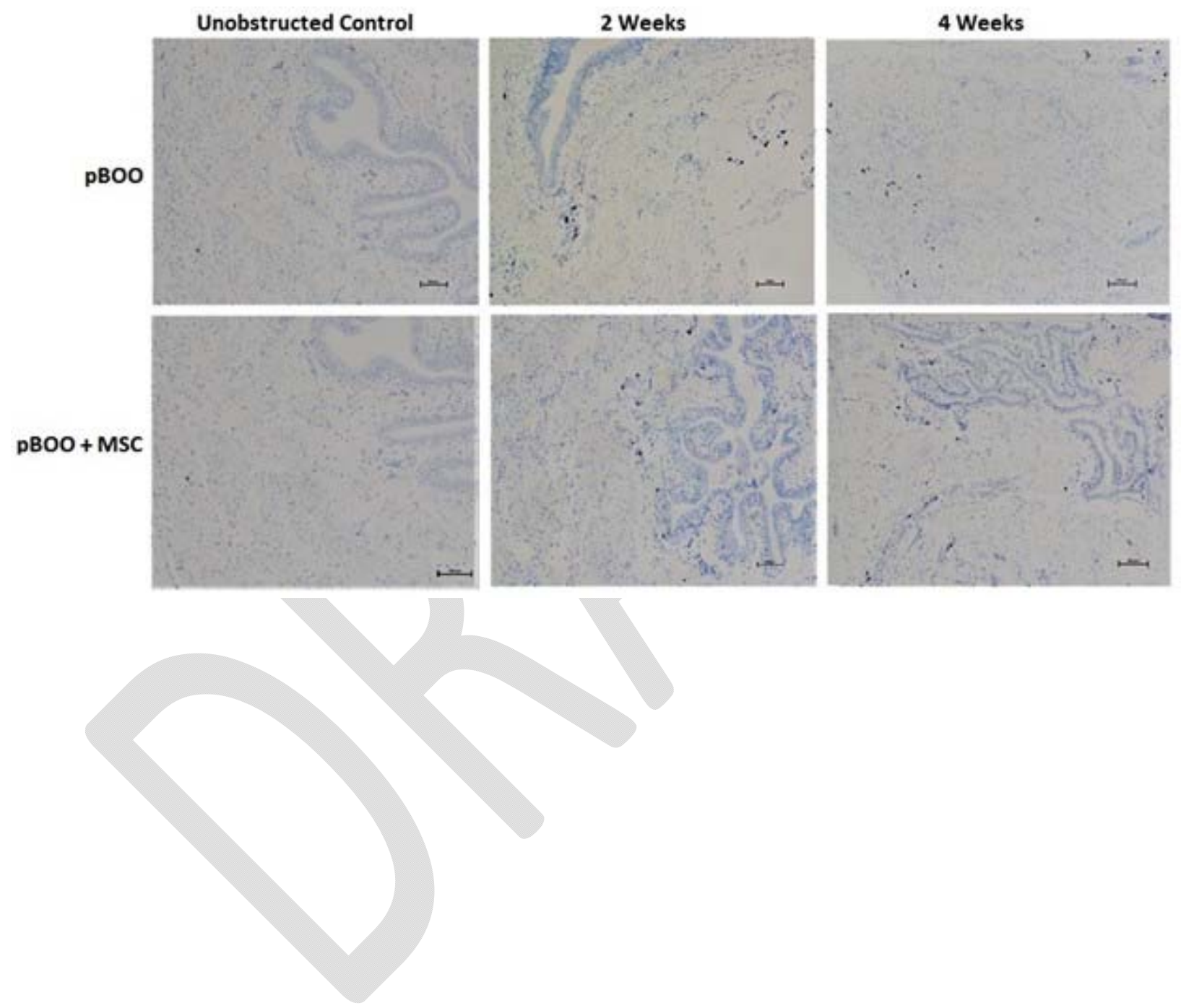


Fig. 3. Mast cell count increased with obstruction time, and degranulated mast cells increased correspondingly. Treatment with mesenchymal stem cells (MSCs) did not affect the total number of mast cell recruitment, nor the rate of degranulation. (A) A representative, nondegranulated mast cell displays a dark violet uniform color and a non-vacant, intact shape. (B) A degranulated mast cell is light purple in color and has dot-like extracellular granules surrounding the deficient cell. (C) The total number of mast cells increased with obstruction with concordant increase in degranulation. "Statistical difference between unobstructed controls and other experimental groups. Both types of mast cells were not significantly affected by MSCs. Micrographs were captured at 40x magnification, and scale bars represent $25 \mu \mathrm{m}$. pBOO: partial bladder outlet obstruction.
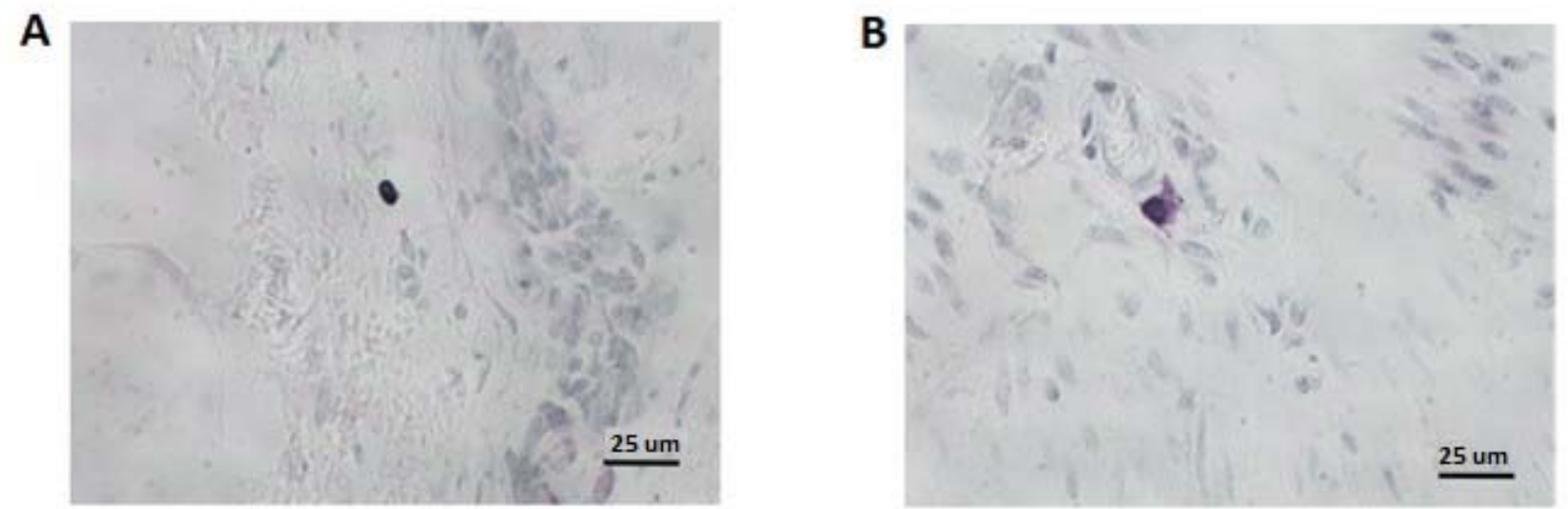

C Number of Mast cells in pBOO \& MSCs Treatment

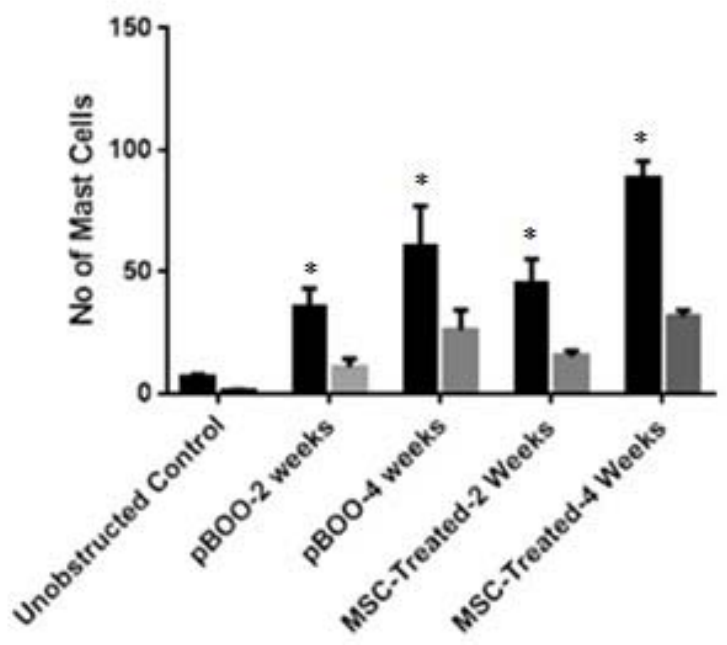


Fig. 4. Collagen expression and infiltration increased in the detrusor and lamina propria layers with obstruction time. However, mesenchymal stem cell (MSC) treatment lead to an inhibition of collagen accumulation at the same time points. Bladder collagen stained blue whereas muscles and blood vessels stained red. Scale bar represents 100 um. pBOO: partial bladder outlet obstruction.

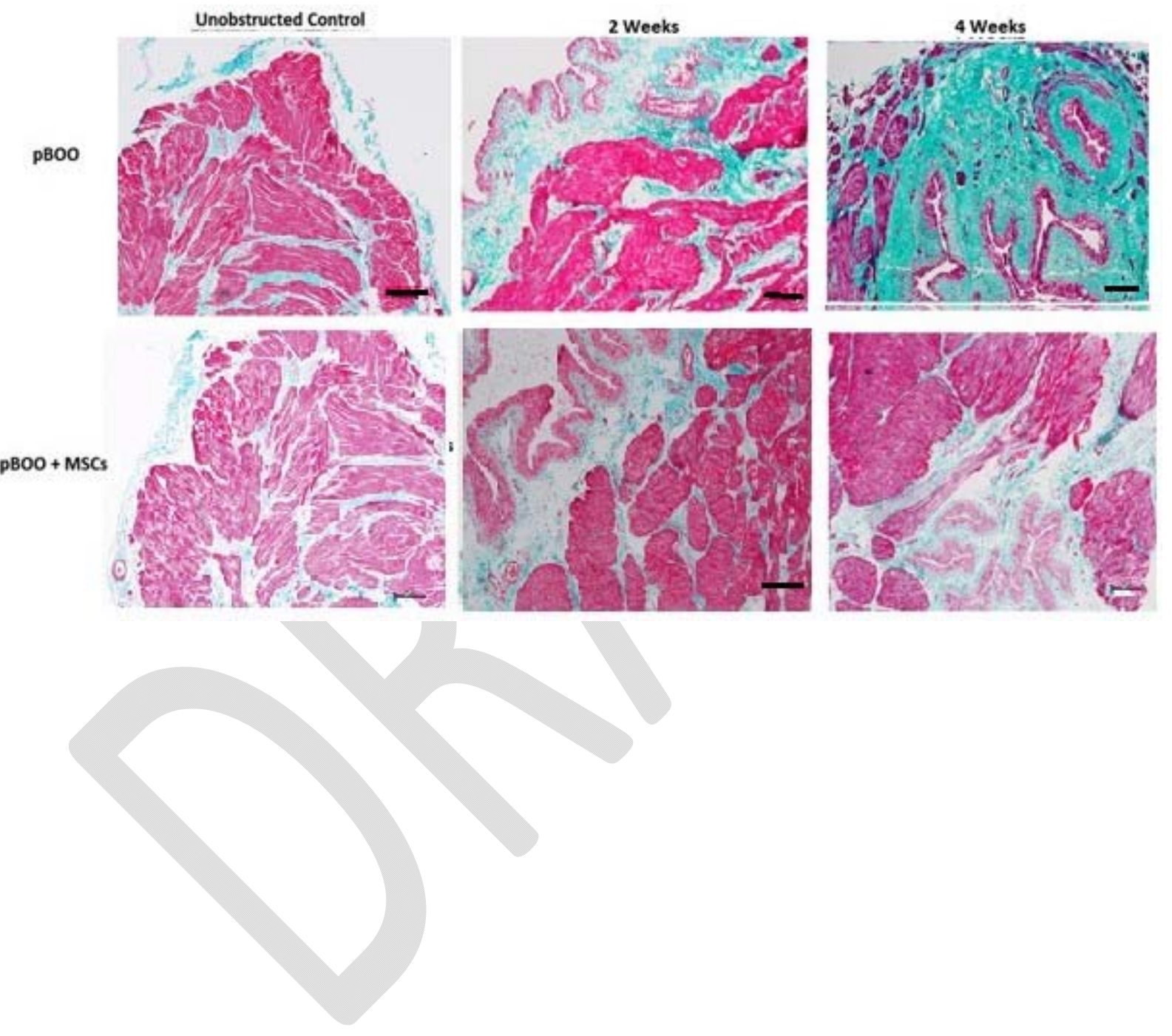

